\title{
NOTE ON SOME BRITISH COLUMBIAN DWARF TREES.
}

\section{(WITH THREE FIGURES)}

While at the Minnesota Seaside Station on the west coast of Vancouver Island during the past summer, an interesting forest of dwarf trees was discovered. For the most part they grew on the weather-worn edges of a strongly inclined slate formation, but a few were found in crevices between blocks of diabase. They were all close to the sea, but outside the influence of the surf. Mr. F. K. Butters and I succeeded in getting a number of

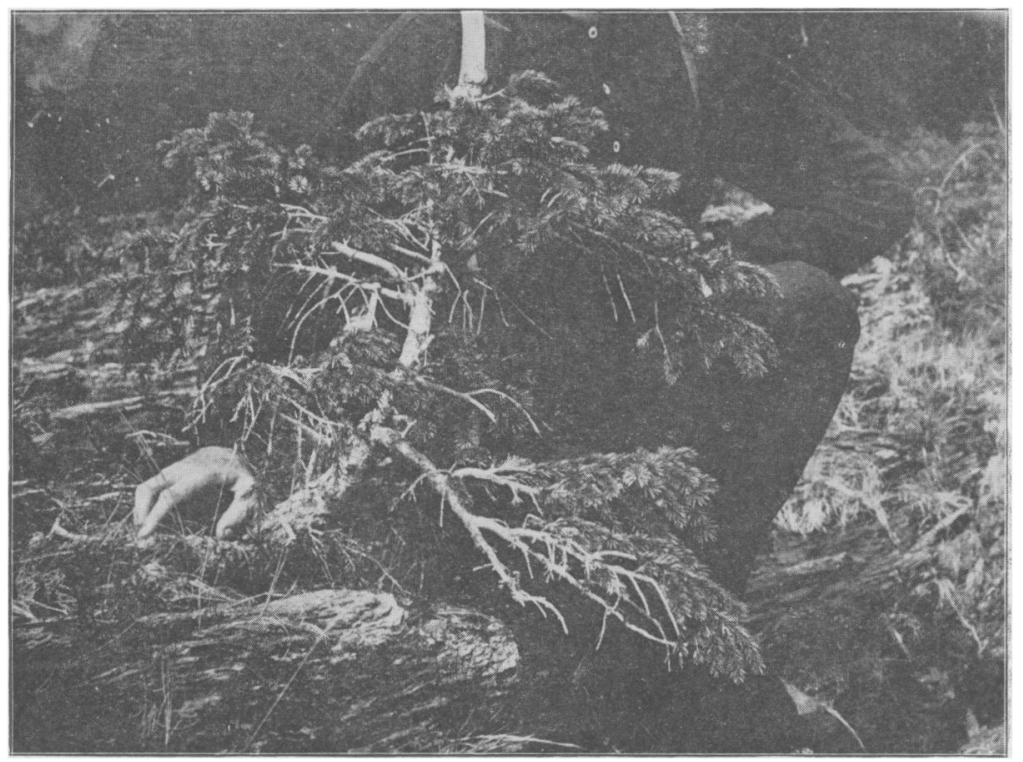

FIG. I.

photographs, after securing which we cut down the trees and determined their age by the he'p of hand lenses and the compound microscope.

The dwarf trees were of three species: Picea sitchensis, Tsuga heterophylla, and Thuja gigantea. The pictures herewith presented, however, are all of the Sitka spruce. Fig. I shows the largest tree photographed and also the youngest. It was a little less than two feet high and sixtyeight years of age. The leaf-bearing phytomeres were decidedly short, but the leaves remain upon the twigs for several years, so that he effect in the picture is of twigs o ord nary length. Fig. 2 shows a tree growing in a 
cleft of the diabase. It was less than a foot in height, with trunk threequarters of an inch in diameter. It turned out to be eighty-six years old. Fig. 3 shows a tree cut down and held in the writer's hand. It was about a foot high, with trunk one inch in diameter, and ninety-eight years old.

These trees have very much the appearance of the well-known Japanese dwarf trees, so much so indeed that it would be an easy matter for the unscrupulous to pot them and palm them off on innocent purchasers. Their striking resemblance to the famous products of Japanese horticultural art suggested to me that from such seashore dwarfs the Japanese might very easily have obtained their hint and learned the tricks of culture.

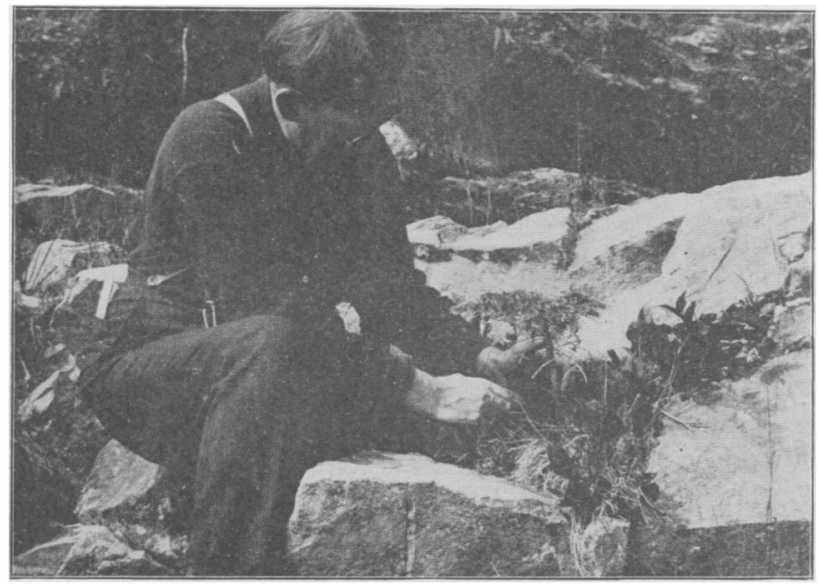

FIG. 2.

Two strong dwarfing influences are at work upon these little British Columbian trees. In the first place, the root system is strongly compressed between plates of rock. Of some of these trees the whole root system was exposed and it looked a good deal like a sheet of brown paper with the trunk of the tree attached to one edge. In the second place, the winds from the sea dwarf and contort the twigs. So with great pressure upon the root system and strong wind action upon the shoot the dwarfing is accomplished.

These little trees have a very different appearance from the slender dwarf spruces of bogs in northern Minnesota. In such regions trees with trunks an inch or so in diameter have been noted, showing an age up to sixty years, but they are tall, slender, and regular in the arrangement 
of their branches. The dwarf trees of mountain tops have likewise a decidedly different appearance, so far as they have come within my observation, and do not particularly resemble the Japanese products.

I do not remember to have seen it suggested anywhere that the dwarf

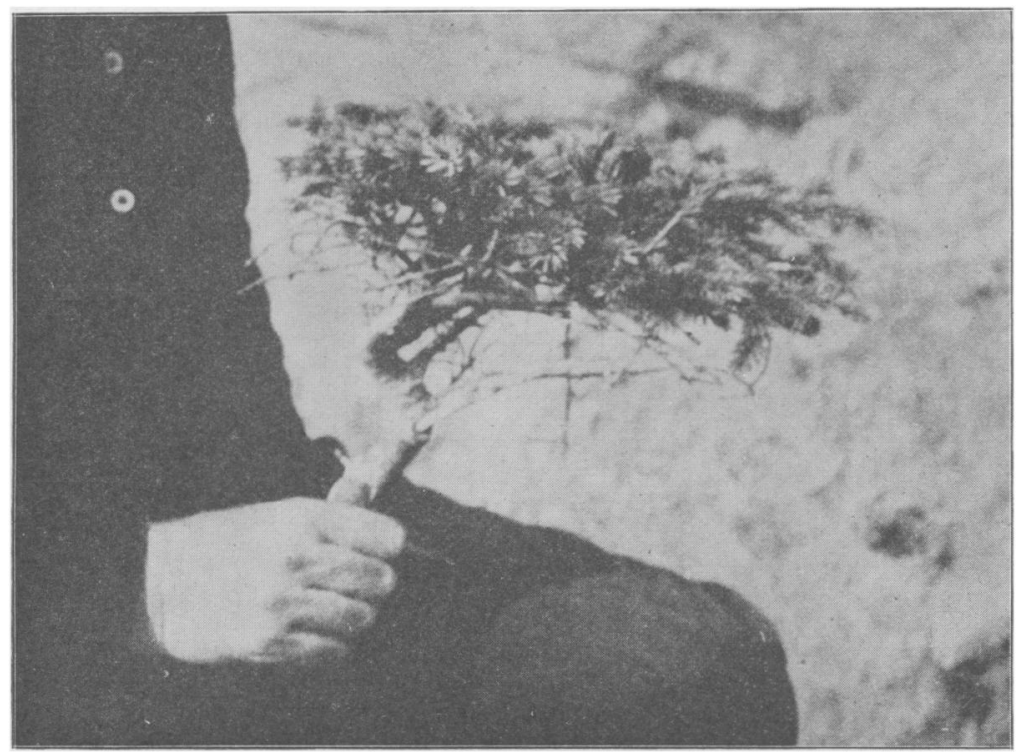

FIG. 3.

trees of Japan are essentially of seashore origin, but in view of the little natural forest on the coast of Vancouver, I think it very possible that this is a correct exp'anation.-Conway MacMillan, University of Minnesota, Minneapolis.

\section{CELLOIDIN TECHNIQUE: A REPLY. .}

In the August number of the Gazette, Dr. Charles J. Chamberlain publishes a criticism of a recent contribution by Mr. A. B. Plowman on the subject of celloidin imbedding. As the account of the method was written up by Mr. Plowman at my suggestion, and the "Correction" of Dr. Chamberlain contains several misconceptions, I think it well to publish a short reply. Dr. Chamberlain is unable to find anything new in the method beyond the preliminary use of hydrofluoric acid. If so accomplished a technician takes the trouble to reperuse the article in question, 